\title{
Imaging Stray Magnetic Field of Individual Ferromagnetic Nanotubes
}

\author{
D. Vasyukov, ${ }^{\dagger, \bigcirc}$ L. Ceccarelli, ${ }^{\dagger, O}$ M. Wyss, ${ }^{\dagger}$ B. Gross, ${ }^{\dagger}$ A. Schwarb, ${ }^{\dagger}$ A. Mehlin, ${ }^{\dagger}$ N. Rossi, ${ }^{\dagger}$ \\ G. Tütüncüoglu, ${ }^{\ddagger}$ F. Heimbach, ${ }^{\S}$ R. R. Zamani, ${ }^{\| \odot ~ A . ~ K o v a ́ c s, ~}{ }^{\perp}$ A. Fontcuberta i Morral, ${ }^{\ddagger \odot}$ D. Grundler, \\ and M. Poggio*,
}

${ }^{\dagger}$ Department of Physics, University of Basel, 4056 Basel, Switzerland

${ }^{\ddagger}$ Laboratory of Semiconductor Materials, Institute of Materials (IMX), School of Engineering, École Polytechnique Fédérale de Lausanne (EPFL), 1015 Lausanne, Switzerland

${ }^{\S}$ Lehrstuhl für Physik funktionaler Schichtsysteme, Physik Department E10, Technische Universität München, 85747 Garching, Germany

"Solid State Physics, Lund University, 22100 Lund, Sweden

${ }^{\perp}$ Ernst Ruska-Centre for Microscopy and Spectroscopy with Electrons, Forschungszentrum Jülich, 52425 Jülich, Germany

\#Laboratory of Nanoscale Magnetic Materials and Magnonics, Institute of Materials (IMX), School of Engineering, École Polytechnique Fédérale de Lausanne (EPFL), 1015 Lausanne, Switzerland

\section{Supporting Information}

ABSTRACT: We use a scanning nanometer-scale superconducting quantum interference device to map the stray magnetic field produced by individual ferromagnetic nanotubes (FNTs) as a function of applied magnetic field. The images are taken as each FNT is led through magnetic reversal and are compared with micromagnetic simulations, which correspond to specific magnetization configurations. In magnetic fields applied perpendicular to the FNT long axis, their magnetization appears to reverse through vortex states, that is, configurations with vortex end domains or in the case of a sufficiently short FNT with a single global vortex. Geometrical imperfections in the samples and the resulting distortion of idealized magnetization configurations influence the measured stray-field patterns.
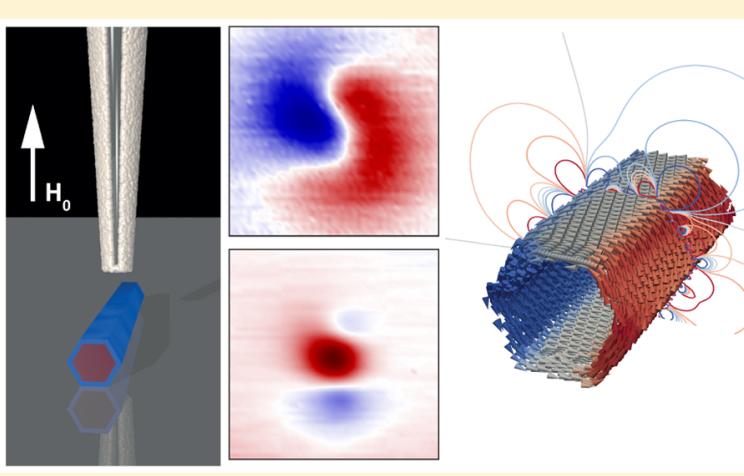

KEYWORDS: Nanomagnetism, nanoscale magnetic imaging, magnetic nanotubes, magnetic tubular architectures, superconducting quantum interference device, SQUID-on-tip

\begin{abstract}
A $s$ the density of magnetic storage technology continues to - grow, engineering magnetic elements with both welldefined remnant states and reproducible reversal processes becomes increasingly challenging. Nanometer-scale magnets have intrinsically large surface-to-volume ratios, making their magnetization configurations especially susceptible to roughness and exterior imperfections. Furthermore, poor control of surface and edge domains can lead to complicated switching processes that are slow and not reproducible. ${ }^{1,2}$

One approach to address these challenges is to use nanomagnets that support remnant flux-closure configurations. The resulting absence of magnetic charge at the surface reduces its role in determining the magnetic state and can yield stable remnant configurations with both fast and reproducible reversal processes. In addition, the lack of stray field produced by fluxclosure configurations suppresses interactions between nearby nanomagnets. Although the stability of such configurations
\end{abstract}

requires dimensions significantly larger than the dipolar exchange length, the absence of dipolar interactions favors closely packed elements and thus high-density arrays. ${ }^{3}$

On the nanometer-scale, core-free geometries such as rings ${ }^{4,5}$ and tubes ${ }^{6}$ have been proposed as hosts of vortex-like fluxclosure configurations with magnetization pointing along their circumference. Such configurations owe their stability to the minimization of magnetostatic energy at the expense of exchange energy. Crucially, the lack of a magnetic core removes the dominant contribution to the exchange energy, which otherwise compromises the stability of vortex states.

Here, we image the stray magnetic field produced by individual ferromagnetic nanotubes (FNTs) as a function of

\footnotetext{
Received: October 14, 2017

Revised: December 21, 2017

Published: January 2, 2018
} 

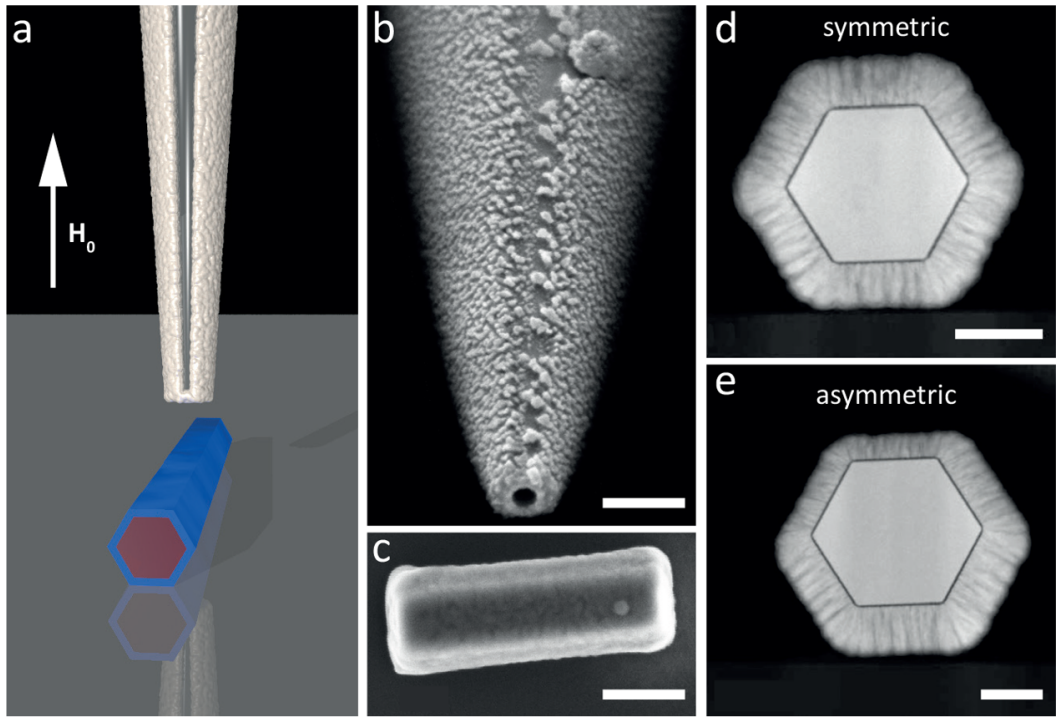

Figure 1. Experimental setup. (a) Schematic drawing showing the scanning SOT, a FNT lying on the substrate, and the direction of $\mathbf{H}_{0}$. The CoFeB shell is depicted in blue and the GaAs core in red. Pb on the SOT is shown in white. SEMs of the (b) the SOT tip and (c) a $0.7 \mu \mathrm{m}$ long FNT. (d,e) Cross-sectional HAADF STEMs of two FNTs from a similar growth batch as those measured. The scalebars represent $200 \mathrm{~nm}$ in $(\mathrm{b}, \mathrm{c}) \mathrm{and} 50 \mathrm{~nm}$ in $(\mathrm{d}, \mathrm{e})$.

applied field using a scanning nanometer-scale superconducting quantum interference device (SQUID). These images show the extent to which flux closure is achieved in FNTs of different lengths as they are driven through magnetic reversal. By comparing the measured stray-field patterns to the results of micromagnetic simulations, we then deduce the progression of magnetization configurations involved in magnetization reversal.

Mapping the magnetic stray field of individual FNTs is challenging due to their small size and correspondingly small magnetic moment. Despite a large number of theoretical studies discussing the configurations supported in FNTs, ${ }^{6-14}$ experimental images of such states have so far been limited in both scope and detail. Cantilever magnetometry, ${ }^{15,16}$ SQUID magnetometry, ${ }^{17,18}$ and magnetotransport measurements ${ }^{19,20}$ have recently shed light on the magnetization reversal process in FNTs, but none of these techniques yield spatial information about the stray field or the configuration of magnetic moments. $\mathrm{Li}$ et al. interpreted the nearly vanishing contrast in a magnetic force microscopy (MFM) image of a single FNT in remnance as an indication of a stable global vortex state, that is, a configuration dominated by a single azimuthally aligned vortex. $^{21}$ Magnetization configurations in rolled-up ferromagnetic membranes between 2 and $16 \mu \mathrm{m}$ in diameter have been imaged using magneto-optical Kerr effect, ${ }^{22} \mathrm{X}$-ray transmission microscopy, ${ }^{22} \mathrm{X}$-ray magnetic circular dichroism photoemission electron microscopy (XMCD-PEEM), ${ }^{23}$ and magnetic soft Xray tomography. ${ }^{24}$ More recently, XMCD-PEEM was used to image magnetization configurations in FNTs of different lengths. $^{25,26}$ Because of technical limitations imposed by the technique, measurement as a function of applied magnetic field was not possible.

We use a scanning SQUID-on-tip (SOT) sensor to map the stray field produced by FNTs as a function of position and applied field. We fabricate the SOT by evaporating $\mathrm{Pb}$ on the apex of a pulled quartz capillary according to a self-aligned method pioneered by Finkler et al. and perfected by Vasyukov et al. ${ }^{27,28}$ The SOT used here has an effective diameter of 150 $\mathrm{nm}$, as extracted from measurements of the critical current $I_{\mathrm{SOT}}$ as a function of a uniform magnetic field $\mathbf{H}_{0}=H_{0} \hat{z}$ applied perpendicular to the SQUID loop. At the operating temperature of $4.2 \mathrm{~K}$, pronounced oscillations of critical current are visible as a function of $H_{0}$ up to $1 \mathrm{~T}$. The SOT is mounted in a custom-built scanning probe microscope operating under vacuum in a ${ }^{4} \mathrm{He}$ cryostat. Maps of the magnetic stray field produced by individual FNTs are made by scanning the FNTs lying on the substrate in the $x y$-plane $300 \mathrm{~nm}$ below the SOT sensor, as shown schematically in Figure 1a. The current response of the sensor is proportional to the magnetic flux threaded through the SQUID loop. For each value of the externally applied field $H_{0}$, a factor is extracted from the current-field interference pattern to convert the measured current $I_{\mathrm{SOT}}$ to the flux. The measured flux then represents the integral of the $z$-component of the total magnetic field over the area of the SQUID loop. By subtracting the contribution of $H_{0}$, we isolate the $z$-component of stray field, $H_{d z}$ integrated over the area of the SOT at each spatial position.

FNT samples consist of a nonmagnetic GaAs core surrounded by a $30 \mathrm{~nm}$-thick magnetic shell of $\mathrm{CoFeB}$ with hexagonal cross-section. $\mathrm{CoFeB}$ is magnetron-sputtered onto template GaAs nanowires (NWs) to produce an amorphous and homogeneous shell, ${ }^{16}$ which is designed to avoid magnetocrystalline anisotropy. ${ }^{29-31}$ Nevertheless, recent magnetotransport experiments show that a small growth-induced magnetic anisotropy may be present. $^{20}$ Scanning electron micrographs (SEMs) of the studied FNTs, as in Figure 1c, reveal continuous and defect-free surfaces, whose roughness is less than $2 \mathrm{~nm}$. Figure 1d,e shows cross-sectional high-angle annular dark-field (HAADF) scanning transmission electron micrographs (STEM) of two FNTs from the same growth batch as those measured, highlighting the possibility for asymmetry due to the deposition process. Dynamic cantilever magnetometry measurements of representative FNTs show $\mu_{0} M_{\mathrm{S}}=1.3 \pm 0.1 \mathrm{~T}^{16}$ where $\mu_{0}$ is the permeability of free space and $M_{\mathrm{S}}$ is the saturation magnetization. Their diameter $d$, which we define as the diameter of the circle circumscribing the hexagonal cross-section, is between 200 and $300 \mathrm{~nm}$. Lengths from 0.7 to $4 \mu \mathrm{m}$ are obtained by cutting individual FNTs into 


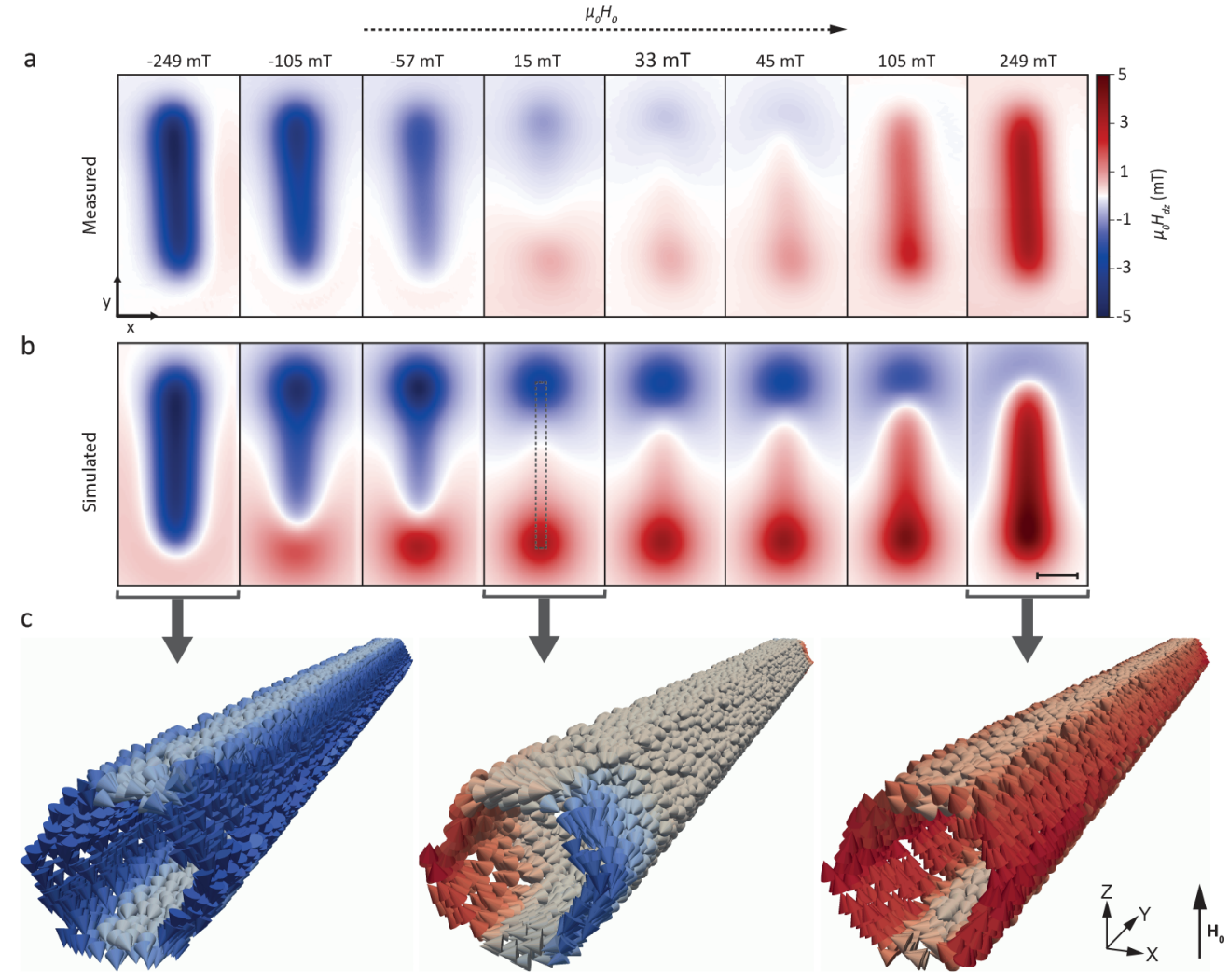

Figure 2. Magnetic reversal of a $4 \mu \mathrm{m}$ long FNT $(l=4.08 \mu \mathrm{m}, d=260 \mathrm{~nm})$ in a field $H_{0}$ applied perpendicular to its long axis. Images of the stray field component along $\hat{z}, H_{d z}$ in the $x y$-plane $300 \mathrm{~nm}$ above the FNT for the labeled values of $\mu_{0} H_{0}$ (a) as measured by the scanning SOT and (b) as generated by numerical simulations of the equilibrium magnetization configuration. The dashed line deliniates the position of the FNT. The scalebar corresponds to $1 \mu \mathrm{m}$. (c) Simulated configurations corresponding to three values of $H_{0}$. The middle configuration, nearest to zero field, shows a mixed state with vortex end domains of opposing circulation sense. Arrows indicate the direction of the magnetization, while red (blue) contrast corresponds to the magnetization component along $\hat{z}(-\hat{z})$.

segments using a focused ion beam (FIB). After cutting, the FNTs are aligned horizontally on a patterned Si substrate. All stray-field progressions are measured as functions of $H_{0}$, which is applied perpendicular to the substrate and therefore perpendicular to the long axes of each FNT. $H_{0}$ is changed at a maximum rate of $8 \mathrm{mT} / \mathrm{s}$. Gross et al. found that similar CoFeB FNTs are fully saturated by a perpendicular field for $\left|\mu_{0} H_{0}\right|>1.2 \mathrm{~T}$ at $T=4.2 \mathrm{~K}^{16}$ Because the serial SQUID array amplifier used in our measurement only allows measurements for $\left|\mu_{0} H_{0}\right| \leq 0.6 \mathrm{~T}$, all the progressions measured here represent minor hysteresis loops.

Figure $2 \mathrm{a}$ shows the stray field maps of a $4-\mu \mathrm{m}$-long FNT for a series of fields as $\mu_{0} H_{0}$ is increased from -0.6 to $0.6 \mathrm{~T}$. The maps reveal a reversal process roughly consistent with a rotation of the net FNT magnetization. At $\mu_{0} H_{0}=-249 \mathrm{mT}$ and at more negative fields, $H_{d z}$ is nearly uniform above the FNT, indicating that its magnetization is initially aligned along the applied field and thus parallel to $-\hat{z}$. As the field is increased toward positive values, maps of $H_{d z}$ show an average magnetization $\langle\mathbf{M}\rangle$, which rotates toward the long axis of the FNT. Near $H_{0}=0$, the two opposing stray field lobes at the ends of the FNT are consistent with an $\langle\mathbf{M}\rangle$ aligned along the long axis. With increasing positive $H_{0}$, the reversal proceeds until the magnetization aligns along $\hat{z}$.

The simulated stray-field maps, shown in Figure $2 b$, are generated by a numerical micromagnetic model of the equilibrium magnetization configurations. We use the software package Mumax $3{ }^{32}$ which employs the Landau-Lifshitz micro- magnetic formalism with finite-difference discretization. The length $l=4.08 \mu \mathrm{m}$ and diameter $d=260 \mathrm{~nm}$ of the FNT are determined by SEMs of the sample, while the thickness $t=30$ $\mathrm{nm}$ is taken from cross-sectional TEMs of samples from the same batch. As shown in Figure 2, the simulated stray-field distributions closely match the measurements. The magnetization configurations extracted from the simulations are nonuniform, as shown in Figure 2c. In the central part of the FNT, the magnetization of the different facets in the hexagonal FNT rotates separately as a function of $H_{0}$, due to their shape anisotropy and their different orientations. As $H_{0}$ approaches zero, vortices nucleate at the FNT ends, resulting in a low-field mixed state, that is, a configuration in which magnetization in the central part of the FNT aligns along its long axis and curls into azimuthally aligned vortex domains at the ends. Experimental evidence for such end vortices has recently been observed by XMCD-PEEM ${ }^{25}$ and $\mathrm{DCM}^{33}$ measurements of similar FNTs at room-temperature. We also measured and simulated a $2 \mu \mathrm{m}$ long FNT of similar cross-sectional dimensions. It shows an analogous progression of stray field maps as a function of $H_{0}$ (see Supporting Information). Simulations suggest a similar progression of magnetization configurations with a mixed state in remnance.

FNTs shorter than $2 \mu \mathrm{m}$ exhibit qualitatively different strayfield progressions. Measurements of a $0.7 \mu \mathrm{m}$ long FNT are shown in Figure 3a. A stray-field pattern with a single lobe persists from large negative field to $\mu_{0} H_{0}=-15 \mathrm{mT}$ without an indication of $\langle\mathbf{M}\rangle$ rotating toward the long axis. Near zero field, 

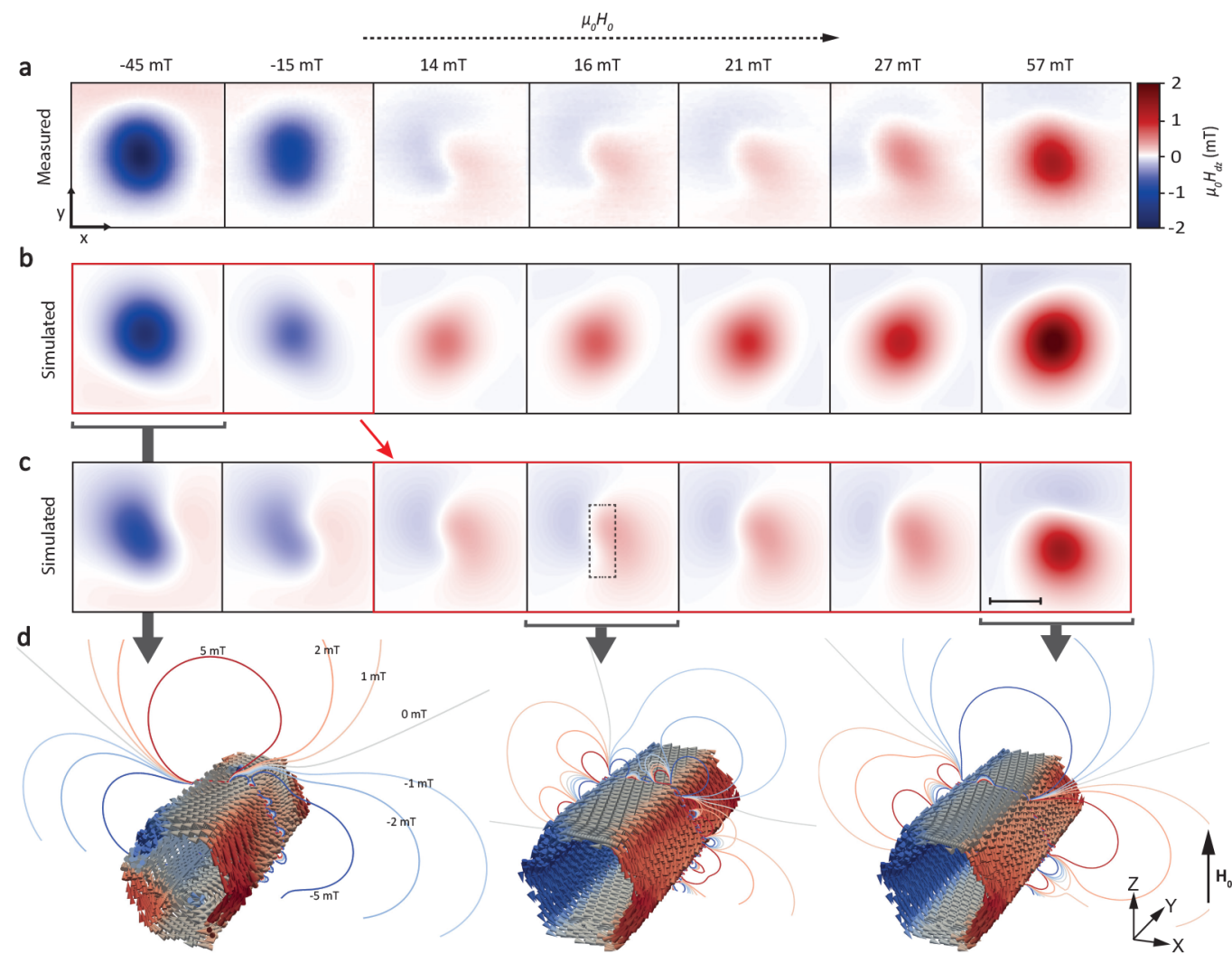

Figure 3. Magnetic reversal of a $0.7 \mu \mathrm{m}$ long FNT $(l=0.69 \mu \mathrm{m}, d=250 \mathrm{~nm})$ in a field applied perpendicular to its long axis. Images of the stray field component along $\hat{z}, H_{d z}$, in the $x y$-plane $300 \mathrm{~nm}$ above the FNT for the labeled values of $H_{0}$ (a) as measured by the scanning SOT. (b,c) Numerical simulations of $H_{d z}$ produced by two progressions of equilibrium magnetization configurations with different initial conditions. The dashed line deliniates the position of the FNT and the scalebar corresponds to $0.5 \mu \mathrm{m}$. (d) Magnetization configurations and contours of constant $H_{d z}$ corresponding to three values of $H_{0}$. The configuration on the left is characterized by two vortices in the top and bottom facets, respectively. The middle and right configurations are distorted global vortex states. Arrows indicate the direction of the magnetization, while red (blue) contrast corresponds to the magnetization component along $\hat{z}(-\hat{z})$.

a stray-field map characterized by an "S"-like zero-field line appears (white contrast in Figure 3a). At more positive fields, a single lobe again dominates. A similar progression of stray field images is also observed upon the reversal of a $1 \mu \mathrm{m}$ long FNT (not shown).

In order to infer the magnetic configuration of the FNT, we simulate its equilibrium configuration as a function of $H_{0}$ using the sample's measured parameters: $l=0.7 \mu \mathrm{m}, d=250 \mathrm{~nm}$, and $t=30 \mathrm{~nm}$. For a perfectly hexagonal FNT with flat ends, the simulated reversal proceeds through different, slightly distorted global vortex states, which depend on the initial conditions of the magnetization. Such simulations do not reproduce the "S"like zero-field line observed in the measured stray-field maps. However, when we consider defects and structural asymmetries likely to be present in the measured FNT, the simulated and measured images come into agreement.

In these refined simulations, we first consider the magnetic "dead-layer" induced by the FIB cutting of the FNT ends as previously reported. ${ }^{34-36}$ We therefore reduce the length of the simulated FNT by $100 \mathrm{~nm}$ on either side. Second, we take into account that the FIB-cut ends of the FNT are not perfectly perpendicular to its long axis. SEMs of the investigated FNT show that the FIB cutting process results in ends slanted by $10^{\circ}$ with respect to $\hat{z}$. Finally, we consider that the $30 \mathrm{~nm}$ thick hexagonal magnetic shell may be asymmetric, that is, slightly thicker on one side of the FNT due to an inhomogeneous deposition, for example, Figure 1e.
With these modifications, the simulated reversal proceeds through at least four different possible stray-field progressions depending on the initial conditions. Only two of these, shown in Figures $3 b, c$, produce stray-field maps which resemble the measurement. The measured stray-field images are consistent with the series shown in Figure $3 \mathrm{~b}$ for negative fields $\left(\mu_{0} H_{0}=\right.$ $-45,-15 \mathrm{mT})$. As the applied field crosses zero $(-15 \mathrm{mT} \leq$ $\left.\mu_{0} H_{0} \leq 14 \mathrm{mT}\right)$, the FNT appears to change stray-field progressions. The images taken at positive fields $(14 \mathrm{mT} \leq$ $\left.\mu_{0} H_{0}\right)$ show patterns consistent with the series shown in Figure $3 c$. The magnetic configurations corresponding to these simulated stray-field maps suggest that the FNT occupies a slightly distorted global vortex state. Before entering this state, for example, at $\mu_{0} H_{0}=-45 \mathrm{mT}$, the simulations show a more complex configuration with magnetic vortices in the top and bottom facets, rather than at the FNT ends. On the other hand, at similar reverse fields, for example, $\mu_{0} H_{0}=57 \mathrm{mT}$, the FNT is shown to occupy a distortion of the global vortex state with an tilt of the magnetization toward the FNT long axis in some of the hexagonal facets.

For some minor loop measurements of short FNTs $(l \leq 1$ $\mu \mathrm{m})$, we obtain stray-field patterns, which the micromagnetic simulations do not reproduce. Two such cases are shown in Figure 4, where (a) represents the stray-field pattern measured above a $0.7 \mu \mathrm{m}$ long FNT at $\mu_{0} H_{0}=20 \mathrm{mT}$ and (d) the pattern measured above a $1 \mu \mathrm{m}$ FNT at $\mu_{0} H_{0}=21 \mathrm{mT}$. Both of these stray-field maps are qualitatively different from the results of 


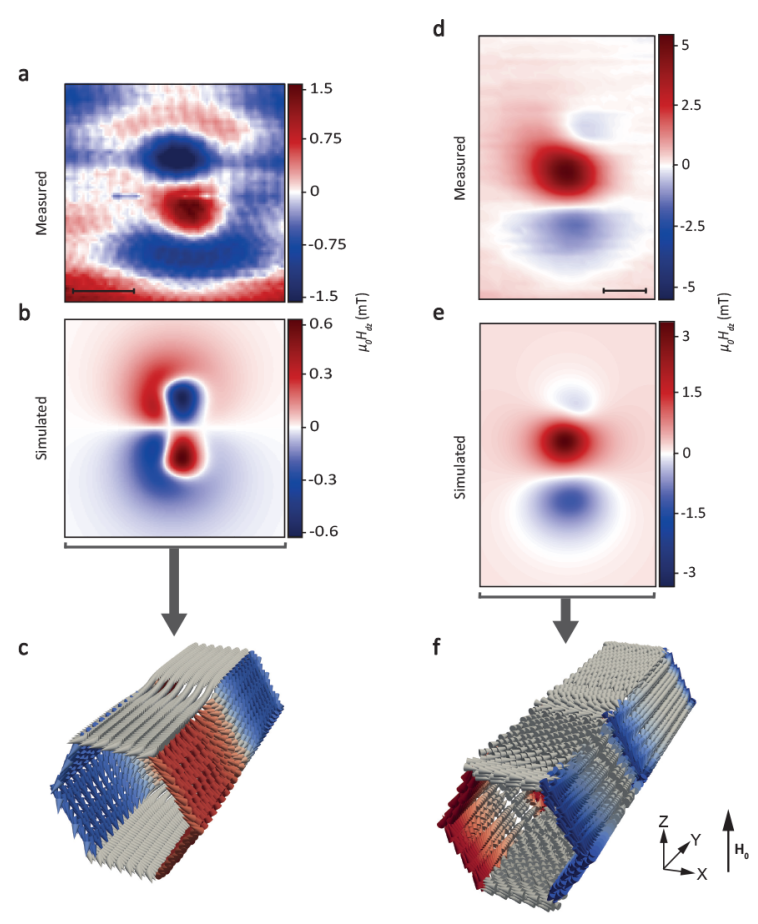

Figure 4. Anomalous stray-field patterns found at low applied field. (a) Stray-field pattern of the $0.7 \mu \mathrm{m}$ long FNT $(l=0.69 \mu \mathrm{m}, d=250 \mathrm{~nm})$ at $\mu_{0} H_{0}=20 \mathrm{mT}$. (b) Similar map produced by an opposing vortex state, shown schematically in (c) and observed near zero field by Wyss et al. ${ }^{25}$ (d) Stray-field pattern of the $1 \mu \mathrm{m}$ long FNT $(l=1.05 \mu \mathrm{m}, d=$ $250 \mathrm{~nm}$ ) at $\mu_{0} H_{0}=21 \mathrm{mT}$. (e) Similar field map produced by a (f) multidomain mixed state with vortex end domains and opposing axial domains separated by a vortex wall. The scalebar corresponds to 0.5 $\mu \mathrm{m}$. In (c,f), arrows indicate the direction of the magnetization, while red (blue) contrast corresponds to the magnetization component along $\hat{z}$.

Figure 3. Because the simulations do not provide equilibrium magnetization configurations that generate these measured stray-field patterns, we test a few idealized configurations in search of possible matches. In particular, the measured pattern shown in Figure 4a is similar to the pattern produced by an opposing vortex state. This configuration, shown in Figure 4c, consists of two vortices of opposing circulation sense, separated by a domain wall. It was observed with XMCD-PEEM to occur in similar-sized FNTs ${ }^{25}$ in remnance at room temperature. The pattern measured in Figure 4e appears to match the stray-field produced by a multidomain state consisting of two head-tohead axial domains separated by a vortex domain wall and capped by two vortex ends, shown in Figure 4f. Although these configurations are not calculated to be equilibrium states for these FNTs in a perpendicular field, they have been suggested as possible intermediate states during reversal of axial magnetization in a longitudinal field. ${ }^{10}$ The presence of these anomalous configurations in our experiments may be due to incomplete magnetization saturation or imperfections not taken into account by our numerical model.

Wyss et al. showed that the types of remnant states that emerge in CoFeB FNTs depend on their length. ${ }^{25}$ For FNTs of these cross-sectional dimensions longer than $2 \mu \mathrm{m}$, the equilibrium remnant state at room temperature is the mixed state, while shorter FNTs favor global or opposing vortex states. Here, we confirm these observations at cyrogenic temperatures by mapping the magnetic stray-field produced by the FNTs rather than their magnetization. In this way, we directly image the defining property of flux-closure configurations, that is, the extent to which their stray field vanishes. In fact, we find that the imperfect geometry of the FNTs causes even the global vortex state to produce stray fields on the order of $100 \mu \mathrm{T}$ at a distance of $300 \mathrm{~nm}$. Finer control of the sample geometry is required in order to reduce this stray field and for such devices to be considered as elements in ultrahigh density magnetic storage. Nevertheless, the global vortex is shown to be robust to the imperfections of real samples; despite slight distortions, it continues to be dominated by a single azimuthally aligned vortex.

Using the scanning SQUID's ability to make images as a function of applied magnetic field, we also reveal the progression of stray-field patterns produced by the FNTs as they reverse their magnetization. Future scanning SOT experiments in parallel applied fields could further test the applicability of established theory to real FNTs. ${ }^{6,10,12,37}$ While the incomplete flux closure and the presence of magnetization configurations not predicted by simulation indicate that FNT samples still cannot be considered ideal, scanning SOT images show the promise of using geometry to program both the overall equilibrium magnetization configurations and the reversal process in nanomagnets.

Methods. SOT Fabrication. SOTs were fabricated according to the technique described by Vasyukov et al. ${ }^{28}$ using a three-step evaporation of $\mathrm{Pb}$ on the apex of a quartz capillary, pulled to achieve the required SOT diameter. The evaporation was performed in a custom-made evaporator with a base pressure of $2 \times 10^{-8}$ mbar and a rotateable sample holder cooled by liquid He. In accordance with Halbertal et al., ${ }^{38}$ an additional Au shunt was deposited close to the tip apex prior to the $\mathrm{Pb}$ evaporation for protection of the SOTs against electrostatic discharge. SOTs were characterized in a test setup prior to their use in the scanning probe microscope.

SOT Positioning and Scanning. Positioning and scanning of the sample below the SOT is carried out using piezo-electric positioners and scanners (Attocube AG). We use the sensitivity of the SOT to both temperature and magnetic field ${ }^{38}$ in combination with electric current, which is passed through a serpentine conductor on the substrate, to position specific FNTs under the SOT (see Supporting Information).

FNT Sample Preparation. The template NWs, onto which the $\mathrm{CoFeB}$ shell is sputtered, are grown by molecular beam epitaxy on a $\mathrm{Si}$ (111) substrate using Ga droplets as catalysts. ${ }^{30}$ During $\mathrm{CoFeB}$ sputter deposition, the wafers of upright and well-separated GaAs NWs are mounted with a $35^{\circ}$ angle between the long axis of the NWs and the deposition direction. The wafers are then continuously rotated in order to achieve a conformal coating. In order to obtain NTs with different lengths and well-defined ends, we cut individual NTs into segments using a Ga FIB in a scanning electron microscope. After cutting, we use an optical microscope equipped with precision micromanipulators to pick up the FNT segments and align them horizontally onto a Si substrate. FNT cross sections for the HAADF STEMs were also prepared using a FIB.

Mumax3 Simulations. To simulate the CoFeB FNTs, we set $\mu_{0} M_{S}$ to its measured value of $1.3 \mathrm{~T}$ and the exchange stiffness to $A_{\mathrm{ex}}=28 \mathrm{pJ} / \mathrm{m}$. The external field is intentionally tilted by $2^{\circ}$ with respect to $\hat{z}$ in both the $x z$ - and the $y z$-plane, in order to exclude numerical artifacts due to symmetry. This angle is within our experimental alignment error. The asymmetry in the magnetic cross-section of an FNT, seen in Figure 1e, is 
generated by removing a hexagonal core from a larger hexagonal wire, whose axis is slightly shifted. In this case, the wire's diameter is $30 \mathrm{~nm}$ larger than the core's diameter and we shift the core's axis below that of the wire by $5 \mathrm{~nm}$. In order to rule out spurious effects due to the discretization of the numerical cells, the cell size must be smaller than the ferromagnetic exchange length of $6.5 \mathrm{~nm}$. This criterion is fulfilled by using a $5 \mathrm{~nm}$ cell size to simulate the $0.7 \mu \mathrm{m}$ long FNT. For the $4 \mu \mathrm{m}$ long FNT, computational limitations force us to set the cell size to $8 \mathrm{~nm}$, such that the full scanning field can be calculated in a reasonable amount of time. Given that the cell size exceeds the exchange length, the results are vulnerable to numerical artifacts. To confirm the reliability of these simulations, we perform a reference simulation with a 4 $\mathrm{nm}$ cell size. Although the magnetic states are essentially unchanged by the difference in cell size, the value of the stray field is altered by up to $10 \%$.

\section{ASSOCIATED CONTENT}

\section{S Supporting Information}

The Supporting Information is available free of charge on the ACS Publications website at DOI: 10.1021/acs.nanolett.7b04386.

Description of the approach and navigation with the SOT aver the FNT samples; discussion of the processing involved in plotting the stray field images taken by the scanning SOT; stray-field images of the magnetic reversal of a $2-\mu \mathrm{m}$-long FNT (PDF)

\section{AUTHOR INFORMATION}

\section{Corresponding Author}

*E-mail: martino.poggio@unibas.ch.

\section{ORCID ${ }^{\circ}$}

R. R. Zamani: 0000-0001-6940-0000

A. Fontcuberta i Morral: 0000-0002-5070-2196

M. Poggio: 0000-0002-5327-051X

\section{Author Contributions}

${ }^{O}$ D.V. and L.C. contributed equally.

\section{Notes}

The authors declare no competing financial interest.

\section{ACKNOWLEDGMENTS}

We thank Jordi Arbiol and Rafal Dunin-Borkowski for work related to TEM, Sascha Martin and the machine shop of the Department of Physics at the University of Basel for technical support, and Ian Dorris for helpful discussions. We acknowledge the support of the Canton Aargau, ERC Starting Grant NWScan (Grant 334767), the SNF under Grant 200020159893, the Swiss Nanoscience Institute, the NCCR Quantum Science and Technology (QSIT), and the DFG Schwerpunkt Programm "Spincaloric transport phenomena" SPP1538 via Project No. GR1640/5-2.

\section{REFERENCES}

(1) Zheng, Y.; Zhu, J.-G. J. Appl. Phys. 1997, 81, 5471.

(2) Fruchart, O.; Nozières, J.-P.; Wernsdorfer, W.; Givord, D.; Rousseaux, F.; Decanini, D. Phys. Rev. Lett. 1999, 82, 1305.

(3) Han, X. F.; Wen, Z. C.; Wei, H. X. J. Appl. Phys. 2008, 103, $07 \mathrm{E} 933$.

(4) Lopez-Diaz, L.; Rothman, J.; Klani, M.; Bland, J. A. C. IEEE Trans. Magn. 2000, 36, 3155.
(5) Rothman, J.; Kläui, M.; Lopez-Diaz, L.; Vaz, C. A. F.; Bleloch, A.; Bland, J. A. C.; Cui, Z.; Speaks, R. Phys. Rev. Lett. 2001, 86, 1098.

(6) Landeros, P.; Suarez, O. J.; Cuchillo, A.; Vargas, P. Phys. Rev. B: Condens. Matter Mater. Phys. 2009, 79, 024404.

(7) Hertel, R.; Kirschner, J. J. Magn. Magn. Mater. 2004, 278, L291.

(8) Escrig, J.; Landeros, P.; Altbir, D.; Vogel, E. E.; Vargas, P. J. Magn. Magn. Mater. 2007, 308, 233.

(9) Escrig, J.; Landeros, P.; Altbir, D.; Vogel, E. E. J. Magn. Magn. Mater. 2007, 310, 2448.

(10) Landeros, P.; Allende, S.; Escrig, J.; Salcedo, E.; Altbir, D.; Vogel, E. E. Appl. Phys. Lett. 2007, 90, 102501.

(11) Chen, A. P.; Guslienko, K. Y.; Gonzalez, J. J. Appl. Phys. 2010, 108, 083920.

(12) Landeros, P.; Núñez, A. S. J. Appl. Phys. 2010, 108, 033917.

(13) Chen, A.-P.; Gonzalez, J. M.; Guslienko, K. Y. J. Appl. Phys. 2011, 109, 073923.

(14) Yan, M.; Andreas, C.; Kákay, A.; García-Sánchez, F.; Hertel, R. Appl. Phys. Lett. 2012, 100, 252401.

(15) Weber, D. P.; Rüffer, D.; Buchter, A.; Xue, F.; Russo-Averchi, E.; Huber, R.; Berberich, P.; Arbiol, J.; Fontcuberta i Morral, A.; Grundler, D.; Poggio, M. Nano Lett. 2012, 12, 6139.

(16) Gross, B.; Weber, D. P.; Rüffer, D.; Buchter, A.; Heimbach, F.; Fontcuberta i Morral, A.; Grundler, D.; Poggio, M. Phys. Rev. B: Condens. Matter Mater. Phys. 2016, 93, 064409.

(17) Buchter, A.; Nagel, J.; Rüffer, D.; Xue, F.; Weber, D. P.; Kieler, O. F.; Weimann, T.; Kohlmann, J.; Zorin, A. B.; Russo-Averchi, E.; Huber, R.; Berberich, P.; Fontcuberta i Morral, A.; Kemmler, M.; Kleiner, R.; Koelle, D.; Grundler, D.; Poggio, M. Phys. Rev. Lett. 2013, 111, 067202.

(18) Buchter, A.; Wölbing, R.; Wyss, M.; Kieler, O. F.; Weimann, T.; Kohlmann, J.; Zorin, A. B.; Rüffer, D.; Matteini, F.; Tütüncüoglu, G.; Heimbach, F.; Kleibert, A.; Fontcuberta i Morral, A.; Grundler, D.; Kleiner, R.; Koelle, D.; Poggio, M. Phys. Rev. B: Condens. Matter Mater. Phys. 2015, 92, 214432.

(19) Rüffer, D.; Huber, R.; Berberich, P.; Albert, S.; Russo-Averchi, E.; Heiss, M.; Arbiol, J.; Fontcuberta i Morral, A.; Grundler, D. Nanoscale 2012, 4, 4989.

(20) Baumgaertl, K.; Heimbach, F.; Maendl, S.; Rueffer, D.; Fontcuberta i Morral, A.; Grundler, D. Appl. Phys. Lett. 2016, 108, 132408.

(21) Li, D.; Thompson, R. S.; Bergmann, G.; Lu, J. G. Adv. Mater. 2008, 20, 4575.

(22) Streubel, R.; Lee, J.; Makarov, D.; Im, M.-Y.; Karnaushenko, D.; Han, L.; Schäfer, R.; Fischer, P.; Kim, S.-K.; Schmidt, O. G. Adv. Mater. 2014, 26, 316.

(23) Streubel, R.; Han, L.; Kronast, F.; Ünal, A. A.; Schmidt, O. G.; Makarov, D. Nano Lett. 2014, 14, 3981.

(24) Streubel, R.; Kronast, F.; Fischer, P.; Parkinson, D.; Schmidt, O. G.; Makarov, D. Nat. Commun. 2015, 6, 7612.

(25) Wyss, M.; Mehlin, A.; Gross, B.; Buchter, A.; Farhan, A.; Buzzi, M.; Kleibert, A.; Tütüncüoglu, G.; Heimbach, F.; Fontcuberta i Morral, A.; Grundler, D.; Poggio, M. Phys. Rev. B: Condens. Matter Mater. Phys. 2017, 96, 024423.

(26) Stano, O. F. 2017, arXiv:1704.06614v1.

(27) Finkler, A.; Segev, Y.; Myasoedov, Y.; Rappaport, M. L.; Ne'eman, L.; Vasyukov, D.; Zeldov, E.; Huber, M. E.; Martin, J.; Yacoby, A. Nano Lett. 2010, 10, 1046.

(28) Vasyukov, D.; Anahory, Y.; Embon, L.; Halbertal, D.; Cuppens, J.; Neeman, L.; Finkler, A.; Segev, Y.; Myasoedov, Y.; Rappaport, M. L.; Huber, M. E.; Zeldov, E. Nat. Nanotechnol. 2013, 8, 639.

(29) Hindmarch, A. T.; Kinane, C. J.; MacKenzie, M.; Chapman, J. N.; Henini, M.; Taylor, D.; Arena, D. A.; Dvorak, J.; Hickey, B. J.; Marrows, C. H. Phys. Rev. Lett. 2008, 100, 117201.

(30) Rüffer, D.; Slot, M.; Huber, R.; Schwarze, T.; Heimbach, F.; Tütüncüoglu, G.; Matteini, F.; Russo-Averchi, E.; Kovács, A.; DuninBorkowski, R.; Zamani, R. R.; Morante, J. R.; Arbiol, J.; Fontcuberta i Morral, A.; Grundler, D. APL Mater. 2014, 2, 076112.

(31) Schwarze, T.; Grundler, D. Appl. Phys. Lett. 2013, 102, 222412. 
(32) Vansteenkiste, A.; Leliaert, J.; Dvornik, M.; Helsen, M.; GarciaSanchez, F.; Van Waeyenberge, B. AIP Adv. 2014, 4, 107133.

(33) Mehlin, A.; Gross, B.; Wyss, M.; Schefer, T.; Tütüncüoglu, G.; Heimbach, F.; Fontcuberta i Morral, A.; Grundler, D.; Poggio, M. arXiv:1711.05164v1, 2017, https://arxiv.org/abs/1711.05164v1.

(34) Katine, J. A.; Ho, M. K.; Ju, Y. S.; Rettner, C. T. Appl. Phys. Lett. 2003, 83, 401.

(35) Khizroev, S.; Litvinov, D. Nanotechnology 2004, 15, R7.

(36) Knutson, C. O. Magnetic domain wall dynamics in nanoscale thin film structures, Ph.D. Thesis, University of Texas, Austin, TX, 2008.

(37) Usov, N. A.; Zhukov, A.; Gonzalez, J. J. Magn. Magn. Mater. 2007, 316, 255.

(38) Halbertal, D.; Cuppens, J.; Shalom, M. B.; Embon, L.; Shadmi, N.; Anahory, Y.; Naren, H. R.; Sarkar, J.; Uri, A.; Ronen, Y.; Myasoedov, Y.; Levitov, L. S.; Joselevich, E.; Geim, A. K.; Zeldov, E. Nature 2016, 539, 407. 